\title{
Autoimmunity after Coronavirus Disease 2019 (COVID-19) Vaccine: A Case of Acquired Hemophilia A
}

\author{
Scott Farley ${ }^{1}$ Robert Ousley ${ }^{1}$ Nicholas Van Wagoner ${ }^{2}$ Fernando Bril ${ }^{1}$ (D) \\ ${ }^{1}$ Internal Medicine, Department of Medicine, University of Alabama \\ at Birmingham, Birmingham, Alabama, United States \\ 2 Division of Infectious Disease, Department of Medicine, University of \\ Address for correspondence Fernando Bril, MD, Internal Medicine, \\ Department of Medicine, University of Alabama at Birmingham, \\ Birmingham, Alabama, United States (e-mail: fbril@uab.edu).
} Alabama at Birmingham, Birmingham, Alabama, United States

Thromb Haemost 2021;121:1674-1676.

Infection with severe acute respiratory syndrome coronavirus 2 (SARS-CoV-2) has been strongly associated with immune-mediated reactions, including the development of autoimmune disorders. ${ }^{1}$ It is theorized that the spike protein S1 of SARS-CoV-2 may be responsible for this phenomenon by means of molecular mimicry. ${ }^{2}$ Emerging data also suggest a link between coronavirus disease 2019 (COVID-19) vaccination and development of various autoimmune conditions ranging from autoimmune hepatitis to immune thrombocytopenia (ITP). ${ }^{3,4}$ This is not a new phenomenon as other vaccines are also associated with immunologic adverse effects, such as Guillain-Barre syndrome, ITP, or vasculitis. ${ }^{5}$

We present a case of acquired hemophilia A (AHA) after application of Pfizer-BioNTech SARS-CoV-2 vaccine. The patient, a 67-year-old African American male with a history of hypertension and asymptomatic pulmonary sarcoidosis not receiving any pharmacological treatment, began to experience left thigh tightness and cramping pain at day +19 after his second dose of the Pfizer-BioNTech COVID-19 vaccine. He originally presented to the emergency department (ED), where an ultrasound was performed, which was negative for deep venous thrombosis. He was instructed to follow up with his sarcoidosis clinic and was discharged from the ED. However, two days later, he presented to another ED for worsening pain and persistent swelling of his left thigh. He was found to have a large hematoma of his left posterior leg extending from his buttock to below his left knee. A smaller area of ecchymosis was also noted on his right arm proximal to the medial epicondyle. Physical exam was otherwise unremarkable. His activated partial thromboplastin time (aPTT) was 72 seconds (normal $25-35$ seconds). Review of prior medical records showed normal aPTT in 2013 and 2014, but no more recent coagulation laboratories. He was transferred to University of Alabama at Birmingham Hospital for further evaluation. His hemoglobin level was $10.2 \mathrm{~g} / \mathrm{dL}$ (from $11.4 \mathrm{~g} / \mathrm{dL} 2$ days prior and down from a baseline of $15.5 \mathrm{~g} / \mathrm{dL}$ in September 2020). A 1:1 mixing study combining the patient's plasma (aPTT of 78 seconds) with a normal control plasma (aPTT of 28 seconds) showed no significant correction of the aPTT (i.e., 60 seconds), suggesting the presence of an inhibitor other than factor deficiency. Factor VIII activity was undetectable (i.e., $<1 \%$ ) and an inhibitor assay confirmed the presence of a factor VIII inhibitor at 110 Bethesda Units $/ \mathrm{mL}$. Other laboratories were significant for: platelets $207,000 / \mathrm{mm}^{3}$, white blood cells $9,740 / \mathrm{mm}^{3}$, prothrombin time 13 seconds (international normalized ratio: 0.99), creatinine $1.27 \mathrm{mg} / \mathrm{dL}$ (estimated glomerular filtration rate $>60 \mathrm{~mL} / \mathrm{min}$ ), blood urea nitrogen $19 \mathrm{mg} / \mathrm{dL}$, aspartate aminotransferase $16 \mathrm{U} / \mathrm{L}$, alanine aminotransferase $19 \mathrm{U} / \mathrm{L}$, total bilirubin $0.6 \mathrm{mg} / \mathrm{dL}$. A computed tomography with intravenous contrast of the chest/abdomen/pelvis from 7 months before presentation showed stable calcified mediastinal and bilateral hilar lymph nodes, without any signs of malignancy. Patient had a colonoscopy in 2017 without any signs of malignancy. Last prostate specific antigen was $0.44 \mathrm{ng} / \mathrm{mL}$ and patient did not report any urinary symptoms. We also obtained antinuclear antibodies, ds-DNA antibodies, and rheumatoid factor, which were negative.

The patient was started on factor eight inhibitor bypass activity (FEIBA) $4,500 \mathrm{u} / \mathrm{kg}$ every 8 hours, oral prednisone $1 \mathrm{mg} / \mathrm{kg}$ daily (i.e., $90 \mathrm{mg}$ daily), and rituximab therapy (375 $\mathrm{mg} / \mathrm{m}^{2}$ weekly for 4 doses). Due to continued hemoglobin drop over the first few days of hospitalization (see - Fig. 1), recombinant factor VIla was also initiated. Seven days after the first dose of rituximab, factor VIII inhibitor decreased to 59 Bethesda Units $/ \mathrm{mL}$, but aPTT persisted in the 70s. Finally, after another 7 days and before the third dose of rituximab, factor VIII inhibitor was 8 Bethesda Units/mL and FEIBA was received

May 27, 2021

accepted

August 3, 2021

published online

August 5, 2021 (c) 2021. Thieme. All rights reserved. Georg Thieme Verlag KG,

Rüdigerstraße 14,

70469 Stuttgart, Germany
DOI https://doi.org/ 10.1055/a-1579-5396. ISSN 0340-6245. 


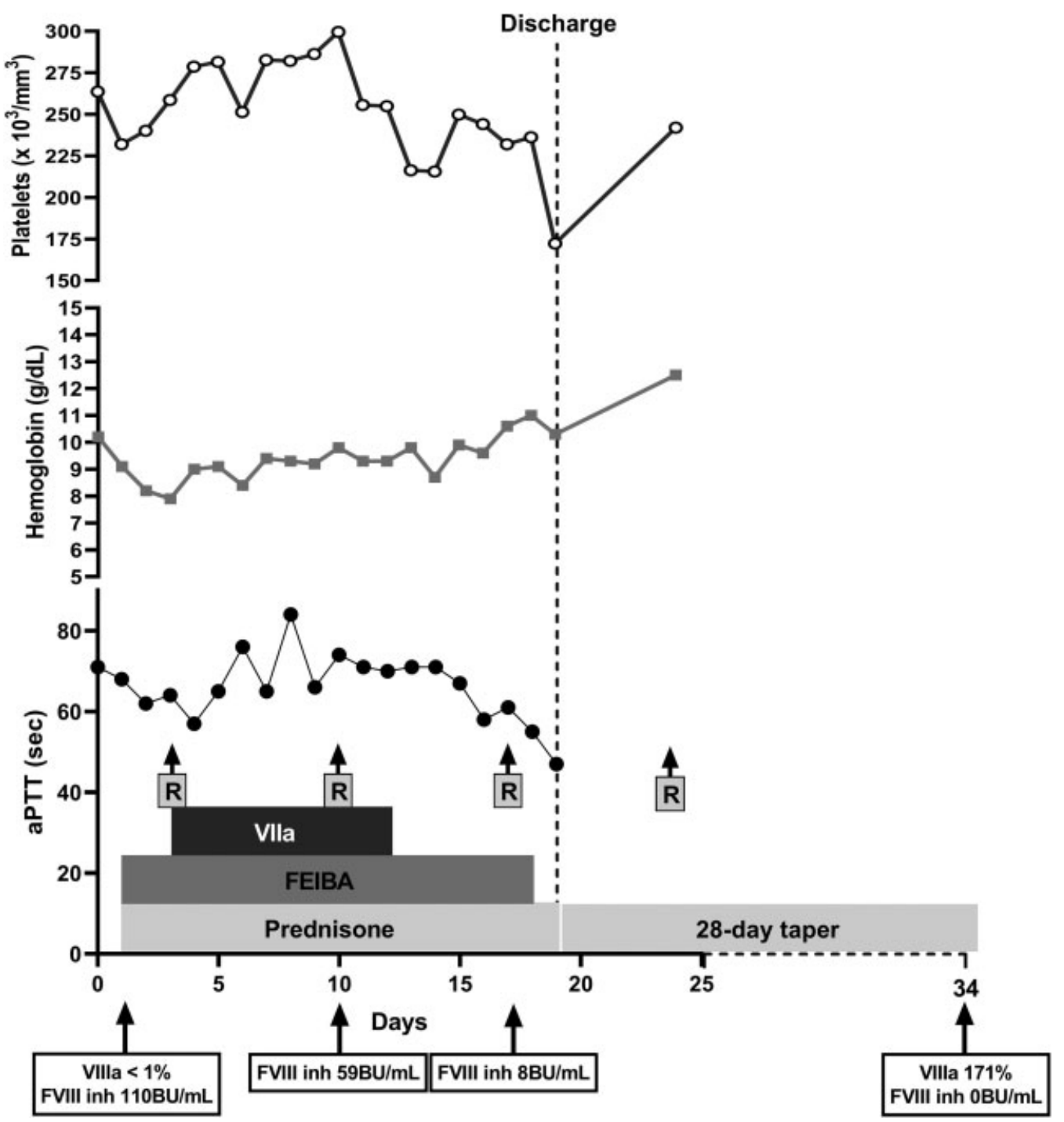

Fig. 1 Changes over time in platelets, hemoglobin, and aPTT. Treatments received as well as factor VIII activity and inhibitor levels were also plotted in the graph. aPTT, activated partial thromboplastin time.

stopped (see - Fig. 1). Patient completed four doses of rituximab and a 28-day taper of prednisone after discharge. Factor VIII activity was at $171 \%$ with undetectable factor VIII inhibitor at day +34 after admission. No new signs of bleeding have been observed after completion of all treatments.

AHA is a rare autoimmune disorder characterized by autoantibody formation against clotting factor VIII, resulting in disruption of secondary hemostasis. Most cases of AHA in nonpregnant and nonpostpartum patients are associated with pre-existing autoimmune conditions or malignancy. ${ }^{6}$ However, this patient had no evidence of malignancy and no pre-existing autoimmune disorders, except for sarcoidosis. No reports of an association between pulmonary sarcoidosis and AHA were found on literature review. While certain medications can be associated with AHA, the patient's medications included only amlodipine, benazepril, pantoprazole, and rizatriptan, none of which have been associated with AHA. $^{7}$ The only identified risk factor for the development of
AHA, based on the suspicious timing, was the Pfizer-BioNTech SARS-CoV-2 vaccine. While the causal relationship cannot be proven, we still felt compelled to report this case to increase awareness among health care providers about potential adverse events after COVID-19 vaccination. Whether the course of AHA after COVID-19 vaccine is similar to regular AHA is also unknown. Unlike prior reports of postCOVID-19 vaccine AHA, ${ }^{8,9}$ our patient had persistence of aPTT elevation with significant hemoglobin drop, requiring a multi-strategy approach with FEIBA, recombinant factor VIIa, glucocorticoids, and rituximab. As we learn more about COVID-19 and the effects of its vaccine, there is an urgent need to report cases like this one, to share our experiences, and help us battle this pandemic.

While SARS-CoV-2 infection has been more frequently associated with a proinflammatory response and prothrombotic complications, ${ }^{10}$ a higher risk of bleeding has also been reported in a subset of patients. ${ }^{11}$ Different immunemediated conditions that predispose to bleeding have also 
been reported during or after COVID-19, such as ITP ${ }^{12}$ and AHA. ${ }^{13,14}$ While the exact mechanisms responsible for these immunohematologic complications are unknown, there is increasing concern that antibodies against SARSCoV-2 spike protein S1 could be responsible for autoimmunity. $^{2}$ In support of this, similar presentations have been reported after COVID-19 vaccination, such as postvaccine ITP $^{4}$ and postvaccine AHA. ${ }^{8}$ Therefore, as COVID-19 infection and current available vaccines only share the $\mathrm{S} 1$ spike protein in common, it is suspected that an immune response against this protein is responsible for autoimmune conditions observed.

We have presented a case of AHA that developed after Pfizer-BioNTech SARS-CoV-2 vaccination. This case adds to the increasing reports of immune-mediated conditions that develop after COVID-19 infection or its vaccine. However, establishing a causal relationship between these vaccines and autoimmune disorders can be challenging due to variable latency periods and diverse presentation of autoimmune processes. ${ }^{9}$ Until more epidemiological and pharmacovigilance information is available, case reports will be crucial to keep health care providers well-informed and on the lookout for these postvaccine complications. Of note, these associations should not discourage patients and physicians from receiving and/or prescribing COVID-19 vaccines, as the potential harm of COVID-19 infection outweighs potential side effects of its vaccines.

\section{Author Contributions}

S.F.: Patient care, writing of the manuscript, and revision of the final version of the manuscript. R.O.: Patient care and revision of the final version of the manuscript. N.V. W.: Patient care and revision of the final version of the manuscript. F.B.: Patient care, editing of the manuscript, and revision of the final version of the manuscript.

\section{Conflict of Interest}

None declared.

\section{References}

1 Ehrenfeld M, Tincani A, Andreoli L, et al. Covid-19 and autoimmunity. Autoimmun Rev 2020;19(08):102597

2 Vojdani A, Kharrazian D. Potential antigenic cross-reactivity between SARS-CoV-2 and human tissue with a possible link to an increase in autoimmune diseases. Clin Immunol 2020; 217:108480

3 Bril F, Al Diffalha S, Dean M, Fettig DM. Autoimmune hepatitis developing after coronavirus disease 2019 (COVID-19) vaccine: causality or casualty? J Hepatol 2021;75(01):222-224

4 Lee EJ, Cines DB, Gernsheimer T, et al. Thrombocytopenia following Pfizer and Moderna SARS-CoV-2 vaccination. Am J Hematol 2021;96(05):534-537

5 Shoenfeld Y, Aron-Maor A. Vaccination and autoimmunity-'vaccinosis': a dangerous liaison? J Autoimmun 2000;14(01):1-10

6 Tiede A, Collins P, Knoebl P, et al. International recommendations on the diagnosis and treatment of acquired hemophilia A. Haematologica 2020;105(07):1791-1801

7 Franchini M, Capra F, Nicolini N, et al. Drug-induced anti-factor VIII antibodies: a systematic review. Med Sci Monit 2007;13(04): RA55-RA61

8 Radwi M, Farsi S. A case report of acquired hemophilia following COVID-19 vaccine. J Thromb Haemost 2021;19(06):1515-1518

9 Cittone MG, Battegay R, Condoluci A, et al. The statistical risk of diagnosing coincidental acquired hemophilia A following antiSARS-CoV-2 vaccination. J Thromb Haemost 2021;19(09): 2360-2362

10 Merad M, Martin JC. Pathological inflammation in patients with COVID-19: a key role for monocytes and macrophages. Nat Rev Immunol 2020;20(06):355-362

11 Zuo Y, Warnock M, Harbaugh A, et al. Plasma tissue plasminogen activator and plasminogen activator inhibitor-1 in hospitalized COVID-19 patients. Sci Rep 2021;11(01):1580

12 Bhattacharjee S, Banerjee M. Immune thrombocytopenia secondary to COVID-19: a systematic review. SN Compr Clin Med 2020. Doi: 10.1007/s42399-020-00521-8

13 Franchini M, Glingani C, De Donno G, et al. The first case of acquired hemophilia A associated with SARS-CoV-2 infection. Am J Hematol 2020;95(08):E197-E198

14 Olsen GM, Rinder HM, Tormey CA. De novo acquired hemophilia as an immune dysregulation phenomenon following SARS-CoV-2 infection. Transfusion 2021;61(03):989-991 